\title{
Effects of Home Laundering on Electrical Resistance of Signal Transmission Lines on Colored E-Textiles
}

\author{
Umut Kivanc Sahin and Senem Kursun Bahadir \\ Department of Textile Engineering, Istanbul Technical University Inonu C. No. 65 34437, Gumussuyu Beyoglu, Istanbul 34437, Turkey
}

Received: January 06, 2017 / Accepted: January 16, 2017 / Published: May 31, 2017.

\begin{abstract}
The aim of this work was to investigate the electrical resistance change of electro-textiles manufactured using cotton fabrics with stainless steel and silver plated PA yarns incorporation after being subjected to home laundering, i.e. detergent washing and silicone softening. Electrical resistances of conductive yarns inside the fabric structure were compared and discussed statistically before and after washing and softener application. Greatest changes in electrical resistances were observed with samples including silver plated PA yarns. After five washing cycles with detergent, silicone softening agent is removed from yarns by washing, and thus conductivity increases. Further washing of e-textiles with detergent for five more cycles causes decrease in conductivity, because of chemical effects of detergent and mechanical effects of washing process such as abrasion due to friction. Detergent which has negative reactive sites bonds with metal ions reduces conductivity.
\end{abstract}

Key words: Conductivity, electrical resistance, signal transmission lines, electro-textiles, conductive yarn, home laundering, detergent, silicone softening agent.

\section{Introduction}

In the last decade, there has been an increasing attraction on use of conductive yarns in textiles in order to develop wearable electronics and functional textiles. Conductive threads are being highly used in order to create functional textile compositions through various conductive pathways that carry information or energy for diverse functional applications, i.e. conductive yarns for electro-textile applications. Among the many studies, some focused on the electro-mechanical performances of conductive yarns while others relate to simple fictionalization of e-textile compositions. The core of some of the investigations focused on feasibility of interconnections for conductive threads purposes [1-6], while others focused on communication systems, several fabrics based antennas operating at $2.45 \mathrm{GHz}$ have been developed using conductive

Corresponding author: Senem Kursun Bahadır, Ph.D., associate professor, research fields: e-textiles, wearable electronics, smart textiles. threads [6-10]. Moreover, there are some other studies focusing on electromagnetic shielding effect of woven e-fabrics containing conductive yarns. In those studies, the effects of varying weft density, warp density, conductive yarn diameter/count, conductive yarn type such as copper, stainless steel, carbon, etc., on electromagnetic shielding effectiveness were studied [11-15]. Apart from weaving techniques, there are other studies where knitting, embroidery, sewing and coating techniques were used to produce e-fabrics with conductive threads [16-21].

Based on the literature review, only few studies were focused on problems encountered during the construction stages or the usage of transmission lines made of conductive yarns. For instance, influence of washing on the electric charge of the coated conductive yarns [22-24], the electrical resistance of textile transmission lines after pre-treatment processes [25], were recently discussed. However, no detailed discussion has yet been made on impact of softener application and detergent washing on the electrical 
resistance of textile transmission lines. In particular, although numerous works were carried out to explain the use of conductive threads in textiles for electro-textile applications, no attempt was made to predict the impact of care processes on electrical resistances of fabrics containing conductive threads.

The softening finishing is one of the most important processes after treatments of textile processes. With chemical softeners, textiles can achieve an agreeable soft hand, some smoothness, more flexibility and better drape and pliability. During preparation, textile materials can become embrittled because natural oils and waxes are removed. Finishing with softeners can overcome this deficiency and even improve the original suppleness (flexibility, pliableness). Other properties improved by softeners include the feeling of added fullness, antistatic properties and sewability. Disadvantages sometimes seen with chemical softeners include reduced crock-fastness, yellowing of white goods, changes in hue of dyed goods and fabric structure slippage. Softeners showed their main effects on the surface of the structure. Small softener molecules, in addition, penetrate the fiber and provide an internal plasticization of the fiber forming polymer by reducing of the glass transition temperature, $T_{\mathrm{g}}$ [26].

The influence of conductive threads on electrical resistance remains a topic for future investigations. Indeed, since conductive threads are composed of metal ions, it is important to know their effect on exposure to wet treatments containing high amounts of ionic chemicals. Thus, in this paper, the changes in electrical resistances of conductive yarns used as transmission lines in cotton fabrics for electro-textile applications were evaluated statistically after softener application and detergent washing processes were completed.

\section{Experimental}

\subsection{Materials}

Since, the applications of e-textiles are so popular for monitoring the human health functions and cotton is friendly for human skin, to produce fabric samples including conductive yarns, $100 \%$ cotton with a yarn count of $20 \mathrm{Ne}$ was considered for this study. They were used as warp and weft yarn in the woven fabric for the nonconductive area in the structure. Conductive yarns were inserted in the weft direction at a defined interval during the design of woven samples (see Fig. 1). The fabric structures including conductive yarns were plain, twill (3/1S) and sateen $(4 / 1)$ weave. The plain fabrics had linear density in warp direction 36 threads/cm and in weft direction 38 threads/cm, whereas twill and sateen fabrics had linear density in warp direction 36 threads/cm and in weft direction 36 threads $/ \mathrm{cm}$. The fabric weights for plain, twill and sateen samples were $124 \mathrm{~g} / \mathrm{m}^{2}, 119 \mathrm{~g} / \mathrm{m}^{2}$, and $115 \mathrm{~g} / \mathrm{m}^{2}$, respectively, the small difference between plain and twill or sateen mainly coming from both construction and linear density of fabrics. Conductive yarns in the structure are positioned in such ways that the signal transmitting for electro-textile applications at a straight trajectory without any undulation is achieved.

The measured characteristics of the conductive yarns used as transmission lines are presented in Table 1.

\subsection{Pretreatment Procedure}

In addition to fabric structure, different fabric pretreatment processes were chosen in order to specify if there is any significant effect on the signal transmitting. The woven fabrics were four grouped

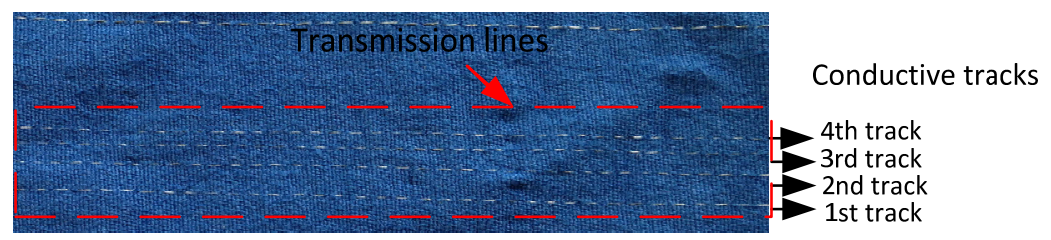

Fig. 1 Transmission lines in a woven fabric sample. 
Table 1 The conductive yarns used as transmission lines.

\begin{tabular}{lllll}
\hline Conductive yarn No. & Material type & Weight $(\mathrm{g} / \mathrm{m})$ & Elongation $(\%)$ & Linear resistance $(\Omega / \mathrm{m})$ \\
\hline 1 & $100 \%$ stainless steel & 0.28 & 1.10 & $<25$ \\
2 & $100 \%$ stainless steel & 0.54 & 1.10 & $<15$ \\
3 & $100 \%$ stainless steel & 1.9 & 1.10 & $<5$ \\
4 & Silver plated PA & 0.125 & 1.10 & $<50$ \\
5 & Silver plated PA & 0.0625 & 1.10 & $<80$ \\
\hline
\end{tabular}

Table 2 Recipes for applied pretreatment processes.

\begin{tabular}{llll}
\hline Treatment process & Recipe & Process temp. $\left({ }^{\circ} \mathrm{C}\right)$ & Process time $(\mathrm{min})$ \\
\hline Desizing & $*$ Amylase, $2 \mathrm{~g} / \mathrm{L}$ & 95 & 45 \\
& $*$ Wetting agent, $2 \mathrm{~g} / \mathrm{L}$ & & \\
& $*$ Wetting agent, $2 \mathrm{~g} / \mathrm{L}$ & 95 & 45 \\
Scouring & $*$ NaOH, $3 \mathrm{~g} / \mathrm{L}$ & & \\
& *Soda, $2 \mathrm{~g} / \mathrm{L}$ & & \\
& $*$ Hydrogen peroxide $\left(\mathrm{H}_{2} \mathrm{O}_{2}\right), 3 \mathrm{~g} / \mathrm{L}$ & 90 & 90 \\
Bleaching & *Peroxide stabilizer, $1 \mathrm{~g} / \mathrm{L}$ & & \\
& *Wetting agent, $1 \mathrm{~g} / \mathrm{L}$ & & \\
& Caustic soda, $3 \mathrm{~g} / \mathrm{L}$ & & \\
\hline
\end{tabular}

and different pretreatment processes were applied to each other. At the beginning, all yarn samples were sized with $100 \%$ starch-sizing agents before weaving process. After weaving, first fabric group was treated with bleaching, second fabric group was treated with desizing + bleaching, third fabric group was treated with scouring + bleaching and fourth fabric group was treated with desizing + scouring + bleaching.

Desizing, scouring and bleaching processes were performed in a jet dyeing machine at a liquor ratio of $25: 1$. The process conditions and ingredients applied to the fabrics are given in Table 2.

All pre-treatment procedures were completed with neutralization and rinse with cold soft water-rinse.

\subsection{Dyeing Profile}

The fabrics were dyed in an ATAC brand lab-type dyeing machine which works at high liquor ratio with, C.I. Reactive Blue 19 (Bezaktiv Blau V-RN 150, supplied by Bezema AG) and C.I. Direct Blue 71 (Tubantin Blau BRR h.c. supplied by Bezema AG).

Reactive Dyeing: The dyebath was based on $1 \mathrm{~g} / \mathrm{L}$ of Meropan DPE (Bezema) and $50 \mathrm{~g} / \mathrm{L}$ sodium chloride dissolved in distilled water at room temperature. The dyeing process commenced as soon as the fabric was added to the dyebath using $60: 1$ liquor-to-fabric weight ratio. After $10 \mathrm{~min}$, the temperature was raised to $60{ }^{\circ} \mathrm{C}$ at a rate of $1.5^{\circ} \mathrm{C} / \mathrm{min}$, and the $5 \mathrm{~g} / \mathrm{L}$ sodium carbonate and 1 $\mathrm{mL} / \mathrm{L}$ of Natronlauge (sodium hydroxide solution) $(32.5 \%)$ were added. The dyeing process was held at this temperature for further $60 \mathrm{~min}$. At the end of the process the fabrics were rinsed with warm and cold water and with $1 \mathrm{~g} / \mathrm{L}$ acetic acid (30\%) neutralized. In order to remove the residual of unfixed dyes and reactants the fabrics were soap-washed at $80{ }^{\circ} \mathrm{C}$ for 20 min in a bath (bath-to fabric weight ratio $60: 1$ ) using $2 \mathrm{~g} / \mathrm{L}$ of non-ionic detergent Cotoblanc NSR (Bezema). The dyeing stage was completed with rinsing with tap water followed by drying at room temperature.

Direct dyeing: The direct dyeing dyebath consists of $0.7 \mathrm{~g} / \mathrm{L}$ leveling agent, Sarabid SBF-N (Bezema), 1 $\mathrm{g} / \mathrm{L}$ sodium carbonate and dyes. The dyeing procedure commenced at $50{ }^{\circ} \mathrm{C}$ using a $70: 1$ liquor-to-fabric weight ratio. Afterwards, the bath was heated-up to $100{ }^{\circ} \mathrm{C}\left(1.5{ }^{\circ} \mathrm{C} / \mathrm{min}\right)$, and $15 \mathrm{~g} / \mathrm{L}$ sodium chloride added after 5 minutes. This temperature was held for $60 \mathrm{~min}$ before the dyebath was cooled down to $50{ }^{\circ} \mathrm{C}$. The rinsing with warm and cold tap water was followed by drying at room temperature.

Here, it should be noted that in order to distinguish 
between mechanical and chemical effects preliminary researches in which the fabrics were run in a mock dye cycle with water only have been conducted. However, preliminary researches have shown that a mock dye cycle with water only does not cause any significant effect on conductivity of transmission lines.

\subsection{Softener Application and Detergent Washing}

The silicone softener used (SETASOFT 1640) was a macro-emulsion of amino-functional silicone with $\mathrm{pH} 5.0-5.5$ in a $10 \%$ solution and complete solubility in water. Softener solutions were prepared at desired $\mathrm{pH}$ with an acetic acid/sodium acetate buffer system, $2 \%$ solutions padded to all colored fabrics at $100 \%$ wet-pick-up, followed by drying at $50{ }^{\circ} \mathrm{C}$.

After silicone softener application, all fabric samples were washed according to BS EN ISO 6330 with a type machine (horizantal axis, front loading), and reference detergent which is non-enzyme, non-phosphate and non-optic. For each washing $20 \mathrm{~g}$ reference detergent was used. Machine settings were arranged as follows: set up time $30 \mathrm{sec}$, set temperature $40{ }^{\circ} \mathrm{C}$ and total time $49 \mathrm{~min}$. Fabrics total weight is $3 \mathrm{~kg}$, because ballast fabric is not used. Thereafter washing, fabrics were dried according to
BS EN ISO 6330 Procedure C-Flat Dry [27]. This washing and drying cycle was repeated ten times and after each five cycles, all fabric samples were re-measured for conductivity.

\subsection{Electrical Resistance Measurement}

The electrical resistance of yarns was measured with Keithley ${ }^{\circledR} 2000$ 61ำ-Digit multimeter. The linear resistance of the conductive yarns is expressed in ohm per meter. Tests were carried out in laboratory conditions of $20 \pm 2{ }^{\circ} \mathrm{C}$ and $65 \pm 2 \%$ relative humidity.

\section{Result and Discussion}

Average linear electrical resistance values after softener and detergent applications are presented in Fig. 2. It is apparent from Fig. 2 that linear resistance values of silver plated PA conductive yarns significantly increase compared to their original (reference) electrical resistance values presented in Table 1, when compared with those of stainless steel yarns. Stainless steel yarns appear to be more resistant to wet chemical processing.

Fig. 3 shows linear resistance values of alternative conductive yarns after being exposed to different pre-treatment processes. It is seen from Fig. 3 that

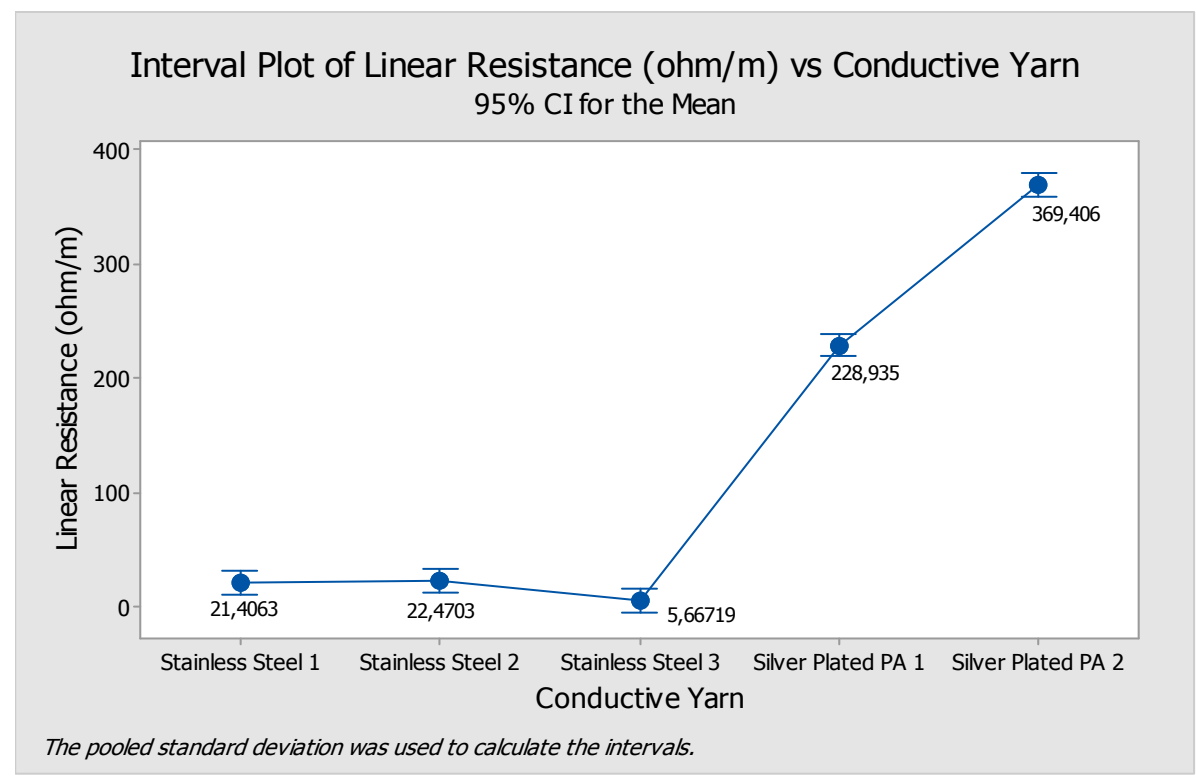

Fig. 2 Interval plot of linear resistance vs. conductive yarns. 


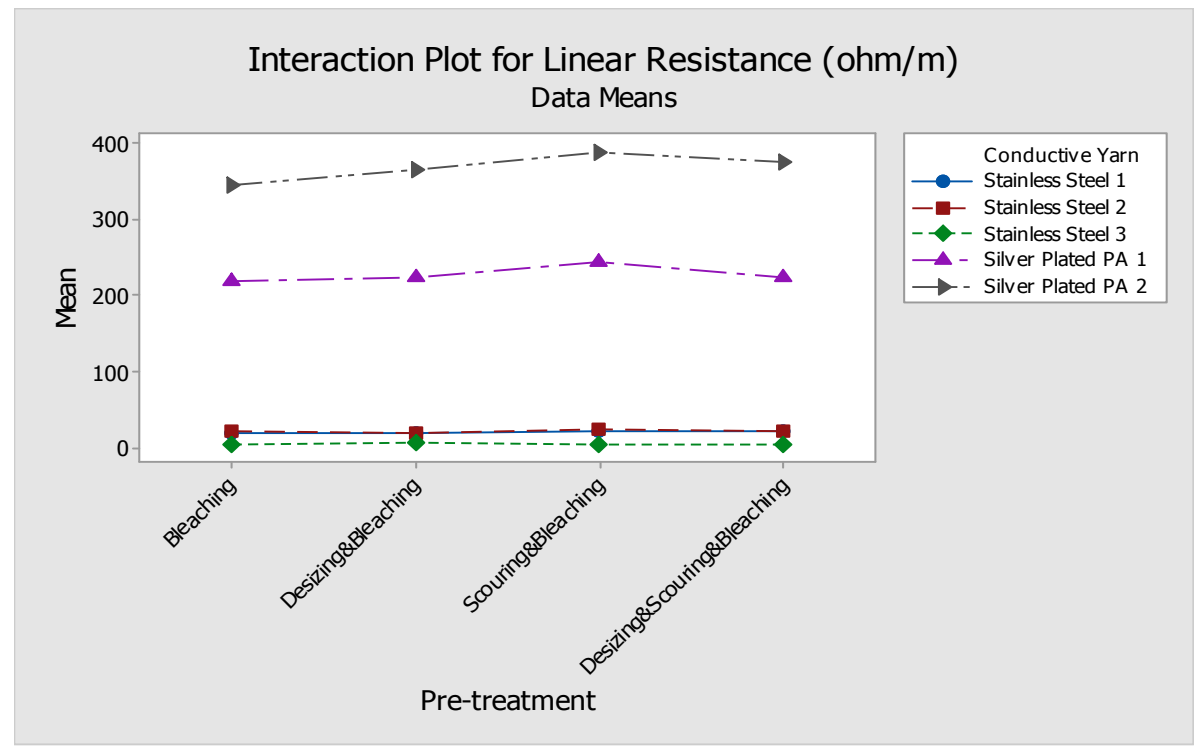

Fig. 3 Interaction plot for linear resistance, pretreatment and conductive yarn.

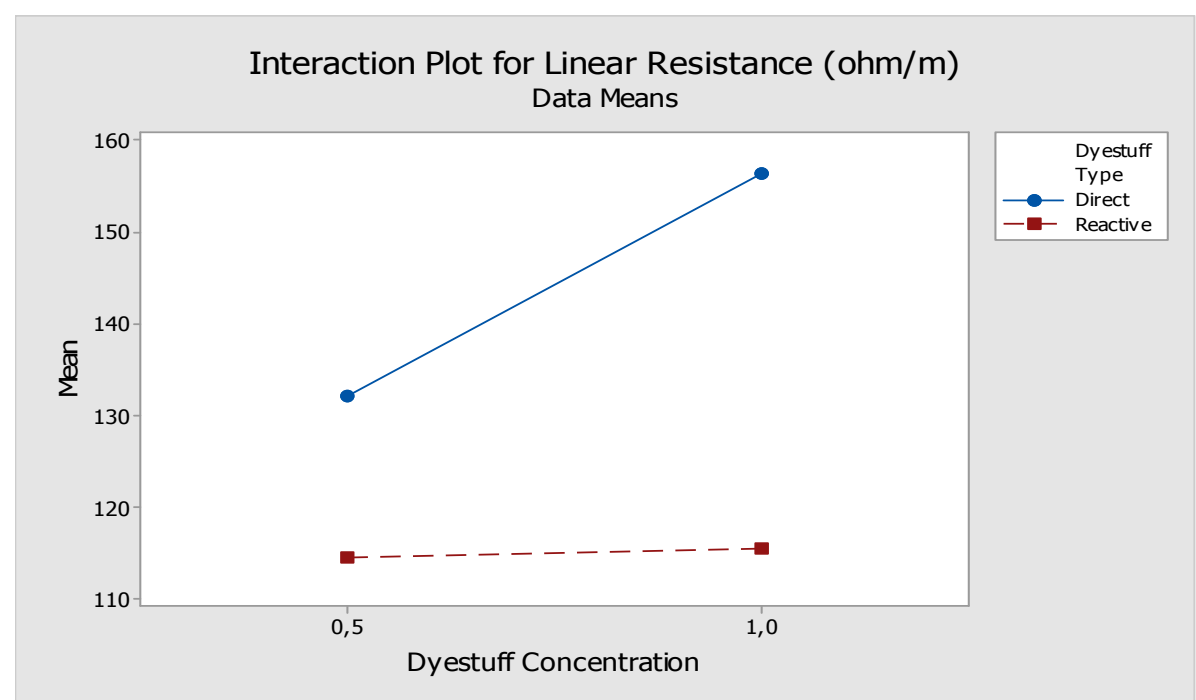

Fig. 4 Interaction plot for linear resistance, dyestuff concentration and dyestuff type.

regardless of the pre-treatment steps, silver plated PA yarns are significantly affected by the chemical wet process, but the effects of the process on $100 \%$ stainless steel yarns are rather limited.

When the single effects of dyestuff type on electrical resistance values are considered (see Fig. 4), it is apparent that direct dyestuff causes a higher increase in electrical resistance, especially when its concentration is increased, compared to that of reactive dyestuff. On the other hand, effect of reactive dyestuff is rather limited, regardless of its concentration. This may be due to the chemical interactions in direct dyeing process and the higher temperature $\left(100{ }^{\circ} \mathrm{C}\right)$ by dyeing with direct dyes. In other words, the ion exchange and easy deformation of conductive tracks which is caused by frictional stresses or chemical interactions in direct dyeing might be responsible for the observed phenomena. Due to ion exchange the electrolyte in dyeing bath may be affected and this may cause an increase in electrical resistance. However, further studies will need to be executed in order to get solid conclusions about the chemical interactions upon the electrical properties. 
Fig. 5 presents single effects of applied wet chemical processes on average linear resistance. The general trend shows that linear resistance of dyed samples increases after softener application, decreases after washing for 5 cycles, but increases back when washing is carried further for five more cycles.

Fig. 6 shows individual effects of applied wet chemical processes on samples comprising different conductive yarns. Detailed investigation on effects of wet chemical processes on individual conductive yarns shows that conductive yarns made of $100 \%$ stainless steel are less prone to being affected by wet chemical processes when compared with silver plated PA yarns. On the other hand, the main effects of applied wet chemical processes are more obvious on silver plated PA yarns.

The gradual increase in electrical resistance values reveals that electrical resistance values generally increase meaning that conductivity of the conductive yarns generally decreases after wet chemical processes. This may be attributed to the mechanical forces involved during wet chemical processes. Mechanical

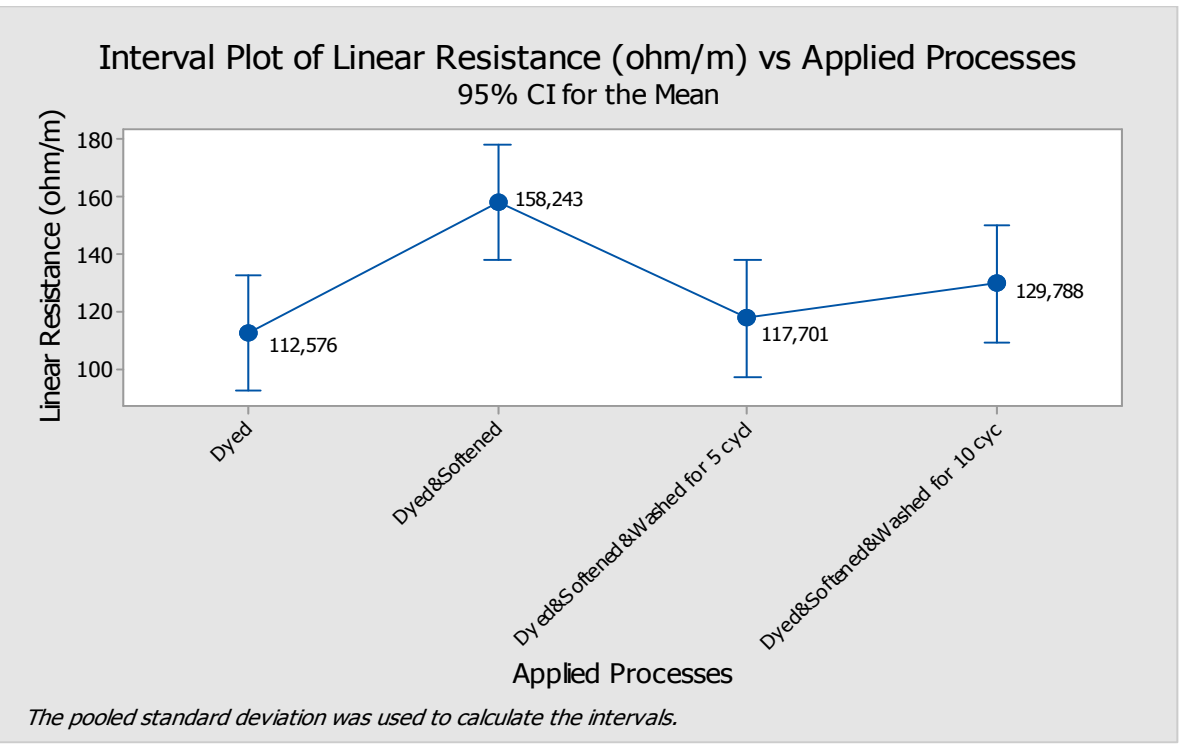

Fig. 5 Interval plot of linear resistance vs. applied processes.

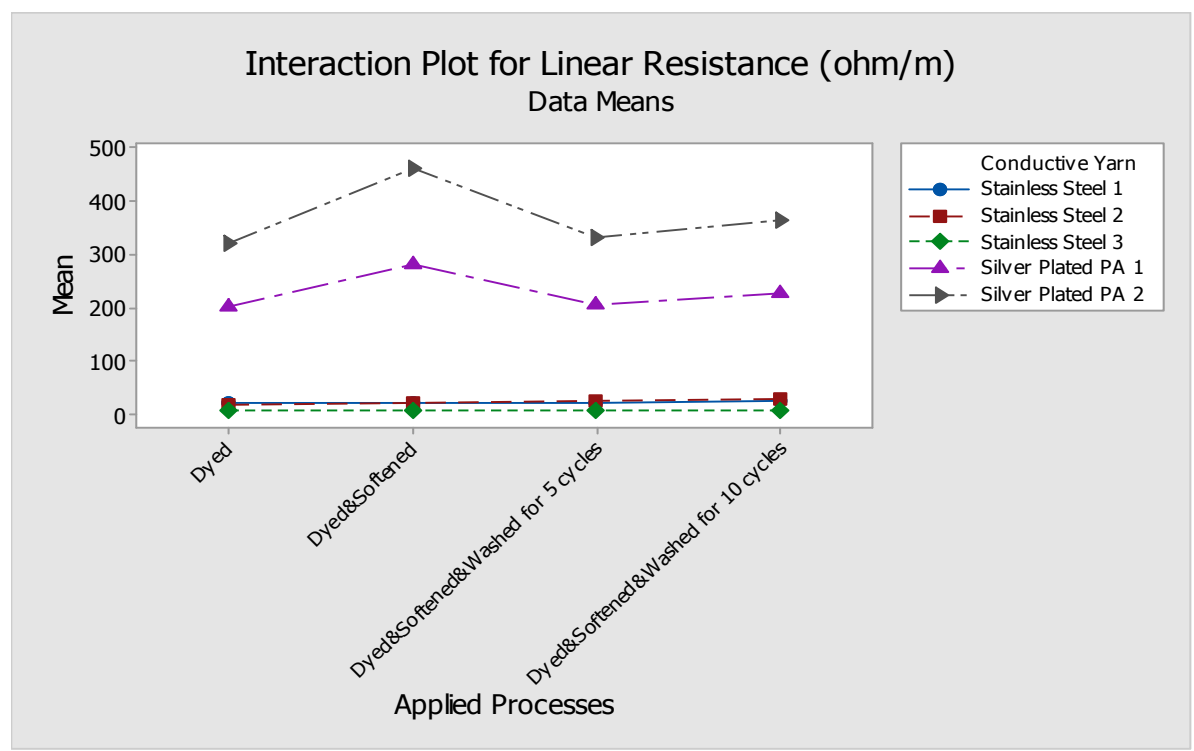

Fig. 6 Interaction plot for linear resistance, applied processes and conductive yarns. 
stresses during softening and detergent washing procedure might lead to the deformation of the surface of the fabric as well as conductive yarns by removing the fibers protruding from the conductive yarns and possibly creating a combing effect. Furthermore, the wet processes may lead to deformation or surface cracks on the conductive yarns passing through fabric structure. From chemical point of view, the high amount of softener and detergent interacts with the conductive yarns. Moreover, use of tap water during dyeing and warm or cold rinsing is believed to cause additional adsorption by cotton of inorganic salt from the hard water and of chemical residues from auxiliaries [28]. All those interactions influence absorption of chemicals by cotton fabric, and deposition of those over conductive yarns, leading to change in their conductivities. The place where deformation occurs is within the individual fibers and conductive yarns, and both are capable of creating movement and damage throughout the woven fabric structures. Additionally, this could possibly leave an impact in surface cracks on the conductive yarns as well as deformation over the transmission lines. The results of this phenomenon may consequently lead to a higher electrical resistance or discontinuities in conductor lines.

\section{Conclusions}

This study was designed to assess the electrical resistances of cotton woven fabrics containing stainless steel and silver plated PA yarns for electro-textile applications. Conventional pre-treatment, direct and reactive dyeing, softening and detergent washing profiles under different concentrations and conditions were adopted to evaluate the change in electrical resistance. The results obtained indicated that all wet chemical processes have considerable impact on electrical resistance of conductive yarns which were used as transmission lines for e-textile applications. It was found that direct dyeing has greater negative impact on electrical resistances of conductive yarns than reactive dyeing. This might be attributed to the differences in the chemistry of direct and reactive dyes and different dyeing process. Additionally, detergent washing and silicone softening were shown to have great influence on electrical resistances of conductive yarns used as transmission lines for electro-textile applications. Greatest changes in electrical resistances were observed with samples including silver plated PA yarns, probably being more prone to being affected by mechanical and chemical impacts due to a two-layered composition. Moreover, silicone softeners having cationic charges are repelled by metallic portions of the yarns, locating themselves on the PA portions through the corroded surface of silver plated yarns, causing decrease in electrons running along the conductive yarn, bringing a decrease to the conductivity of the yarn. On the other hand, after five washing cycles with detergent, silicone softening agent is removed from yarns by washing, and thus conductivity increases. Further washing of e-textiles with detergent for five more cycles causes decrease in conductivity, because of chemical effects of detergent and mechanical effects of washing process such as abrasion due to friction. Detergents have negative reactive sites that bond with metal ions and reduce conductivity.

\section{Acknowledgment}

This project was supported by ITU-BAP Project No. 36120. Grateful appreciation is extended to Istanbul Technical University, Textile Clothing Control and the Research Laboratory; and special gratitude to Mustafa Yildirim and Hamdi Demirler for their support in the supply of materials and performing experimental work.

\section{References}

[1] Li, L., Liu, S., Ding, F., Hua, T., Au, W. M., and Wong, K. S. 2012. "Electromechanical Analysis of Length-related Resistance and Contact Resistance of Conductive Knitted Fabrics." Textile Research Journal 82 (20): 2062-70. 
[2] Dhawan, A. 2007. "Development of Robust Fiber Optic Sensors Suitable for Incorporation into Textiles, and a Mechanical Analysis of Electronic Textile Circuits." Ph.D. thesis, North Carolina State University.

[3] Dhawan, A., Seyam, A. M., Ghosh, T. K., and Muth, J. F. 2004. "Woven Fabric-Based Electrical Circuits Part I: Evaluating Interconnect Methods." Textile Research Journal 74 (10): 913-9.

[4] Dhawan, A., Tushar, K., Ghosh, T. K., Abdelfattah, M., and Seyam, A. M. 2010. "Woven Fabric-Based Electrical Circuits Part II: Yarn and Fabric Structures to Reduce Crosstalk Noise in Woven Fabric-Based Circuits." Textile Research Journal 74 (11): 955-60.

[5] Locher, I. 2010. "Enabling Technologies for Electrical Circuits on a Woven Monofilament Hybrid Fabric." Textile Research Journal 78 (7): 583-94.

[6] Visser, H. J., and Reniers, C. F. 2007. "Textile Antennas, a Practical Approach.” In Proceedings of the IET Seminar Digests, 538-8.

[7] Sanz-Izquierdo, B., and Batchelor, J. C. 2008. "A Dual Band Belt Antenna." International Workshop on Antenna Technology: Small Antennas and Novel Metamaterials, 374-7.

[8] Salonen, P., and Rahmat-Samii, Y. 2006. "Textile Antennas: Effects of Antenna Bending on Input Matching and Impedance Bandwidth." In Proceedings of the First European Conference on Antennas and Propagation, 1-5.

[9] Salonen, P., and Keskilammi, M. 2008. "SoftWear Antenna." In Proceedings of the IEEE Military Communications Conference, 1-6.

[10] Nakad, Z., Jones, M., Martin, T., and Shenoy, R. 2007. "Using Electronic Textiles to Implement an Acoustic Beamforming Array: A Case Study." Pervasive and Mobile Computing 3 (5): 581-606.

[11] Cheng, K. B. 2006. "Electromagnetic Shielding Effectiveness of the Twill Copper Woven Fabrics." Journal of Reinforced Plastics and Composites 25 (7): 699-709.

[12] Roh, J. S., Chi, Y. S., Kang, T. J., and Nam, S. W. 2008. "Electromagnetic Shielding Effectiveness of Multifunctional Metal Composite Fabrics." Textile Research Journal 78 (9): 825-35.

[13] Lee, S. E., Park, K. Y., Oh, K. S., and Kim, C. G. 2009. "The Use of Carbon/Dielectric Fiber Woven Fabrics as Filters for Electromagnetic Radiation.” Carbon 47 (8): 1896-904.

[14] Kayacan, O., and Bulgun, E. Y. 2009. "Heating Behaviors of Metallic Textile Structures." International Journal of Clothing Science and Technology 21 (2/3): 127-36.

[15] Kayacan, O., Bulgun, E. Y., and Sahin, O. 2009. "Implementation of Steel-Based Fabric Panels in a
Heated Garment Design.” Text. Res. J. 79 (16): 1427-37.

[16] Wan, K. M. 2010. "A Resistive Network Model for Conductive Knitting Stitches Resistive Network of Knitting Stitches." Textile Research Journal 80 (10): $1-13$.

[17] Li, L., Au, W. M., Li, Y., Wan, K. M., Chung, W. Y., and Wong, K. S. 2009. "A Novel Design Method for Intelligent Clothing Based on Garment Design and Knitting Technology." Textile Research Journal 79 (18): 1670-9.

[18] Linz, T., Kallmayer, C., Aschenbrenner, R., and Reichl, H. 2005. "Embroidering Electrical Interconnects with Conductive Yarn for the Integration of Flexible Electronic Modules into Fabric." In Proceedings of the Ninth IEEE International Symposium on Wearable Computers, 86-91.

[19] Linz, T., Gourmelon, L., and Langereis, G. 2007. "Contactless EMG Sensors Embroidered onto Textile." In Proceedings of the 4th International Workshop on Wearable and Implantable Body Sensor Networks, 13: 29-34.

[20] Odhiambo, S. A., Gilbert, D. M., Hertleer, C., Schwarz, A., and Langenhove, L. V. 2014. "Discharge Characteristics of Poly(3,4-ethylene dioxythiophene) : Poly (styrenesulfonate) (PEDOT : PSS) Textile Batteries; Comparison of Silver Coated Yarn Electrode Devices and Pure Stainless Steel Filament Yarn Electrode Devices." Textile Research Journal 84 (4): 347-54.

[21] Alagirusamy, R., Eichhoff, J., Gries, T., and Jockenhoevel, S. 2013. "Coating of Conductive Yarns for Electro-textile Applications." Journal of the Textile Institute 104 (3): 270-7.

[22] Varnaite, S., and Katunskis, J. 2009. "Influence of Washing on the Electric Charge Decay of Fabrics with Conductive Yarns." Fibres \& Textiles in Eastern Europe 17 (5): 69-75.

[23] Varnaitè, S., Vitkauskas, A., Abraitienè, A., Rubežienè, V., and Valienè, V. 2008. "The Features of Electric Charge Decay in the Polyester Fabric Containing Metal Fibres." Journal of Materials Science: Materials in Medicine 14 (2): 157-61.

[24] Schwarz, A., Hakuzimana, J., Westbroek, P., Gilbert, D. M., Priniotakis, G., Nyokong, T., and Langenhove, V. L. 2012. "A Study on the Morphology of Thin Copper Films on Paraaramid Yarns and Their Influence on the Yarn's Electro-conductive and Mechanical Properties." Textile Research Journal 82 (15): 1587-96.

[25] Kursun-Bahadir, S. 2015. "The Effect of Textile Pretreatment Processes on Signal Transferring Capability of Textile Transmission Lines." Fibres \& Textiles in Eastern Europe 23 (2/110): 55-62.

[26] Schindler, W. D., and Hauser, P. J. 2004. Chemical 


\section{Lines on Colored E-Textiles}

Finishing of Textiles. 1st ed. Cambrdige, England: Woodhead Publishing Ltd., 29-38.

[27] BS EN ISO 6330: 2012. Textiles-Domestic Washing and Drying Procedures for Textile Testing.
[28] Carrión, F. J. 1994. "The Influence of the Temperature on the Sorption of Microemulsionated Cationic Softeners on Cotton." Journal of the Society of Dyers and Colourists 110 (7): 234-7. 